\title{
Characterization of thermal neutron beam monitors
}

\author{
F. Issa, ${ }^{*}$ A. Khaplanov, and R. Hall-Wilton ${ }^{\dagger}$ \\ European Spallation Source ESS ERIC, P.O Box 176, SE-221 00 Lund, Sweden \\ I. Llamas, M. Dalseth Riktor, and S. R. Brattheim \\ Institute for Energy Technology, P.O. Box 40 Kjeller, Norway \\ H. Perrey ${ }^{3}$ \\ Lund University, P.O Box 118, SE-221 00 Lund, Sweden and European Spallation Source ESS ERIC, \\ P.O Box 176, SE-221 00 Lund, Sweden \\ (Received 9 February 2017; published 26 September 2017)

\begin{abstract}
Neutron beam monitors with a wide range of efficiencies, low $\gamma$ sensitivity, and high time and space resolution are required in neutron beam experiments to continuously diagnose the delivered beam. In this work, commercially available neutron beam monitors have been characterized using the R2D2 beamline at IFE (Norway) and using a Be-based neutron source. For the $\gamma$ sensitivity measurements different $\gamma$ sources have been used. The evaluation of the monitors includes, the study of their efficiency, attenuation, scattering, and sensitivity to $\gamma$. In this work we report the results of this characterization.
\end{abstract}

DOI: 10.1103/PhysRevAccelBeams.20.092801

\section{INTRODUCTION}

Neutron scattering is used in modern science to understand material properties on the atomic scale. It has led to advances in many areas of science, from clean energy to nanotechnology, materials engineering, and fundamental physics. High-intensity beams of neutrons are needed to perform neutron scattering science. These neutron beams can be produced either by fission in a nuclear reactor, similar to that at the Institut Laue-Langevin (ILL) [1], or by spallation sources such as at ISIS [2], SNS [3], and J-PARC [4]. The European Spallation Source (ESS) [5,6] which is under construction in Sweden, will be the world's leading neutron source for the studies of materials. A unique feature of the ESS neutron production will be the long proton accelerator pulse $(2.86 \mathrm{~ms})$ with a repetition rate of $14 \mathrm{~Hz}$ [7].

At a neutron spallation source, the neutron intensity fluctuates with time depending on the characteristics of the proton beam to the neutron target and moderator performance. This requires a continuous neutron beam monitoring with high precision to ensure the correct operation of the neutron instruments. More specifically a continuous

\footnotetext{
*fatima.issa@esss.se

Also at Mid-Sweden University, SE-851 70 Sundsvall, Sweden.

Published by the American Physical Society under the terms of the Creative Commons Attribution 4.0 International license. Further distribution of this work must maintain attribution to the author(s) and the published article's title, journal citation, and DOI.
}

monitoring of neutrons in the subpulse structure is required for instruments with time-of-flight design.

Neutron beam monitors are detectors with sufficiently low efficiency $\left(10^{-6}-10^{-1}\right)$ so that a low percentage of the incoming beam is absorbed or scattered. They are used to ensure that the neutron flux, beam distribution, and pulse timing correspond to those expected from the design of the instrument. In addition, they are used to determine the neutron flux at the sample in order to correctly interpret the scattering data.

Given the diversity of the neutron instruments a variety of neutron beam monitors will be required. Multiwire proportional counters (MWPC) have been used in many facilities as neutron beam monitors for instruments with moderate flux and low count rate requirement. They can be filled with either ${ }^{3} \mathrm{He}$ at low pressure or nitrogen as the sensing gas. For cases where a high-count rate is needed, such as for profiling on pulse-by-pulse basis, monitors based on gas electron multipliers (GEM) are more appropriate. Both types of monitors can be equipped with position sensitive readout in either 1 or 2 dimensions. Scintillation monitors are also an option for low count rate situations. Fission chambers have an advantage of a very high neutron signal and therefore a very strong $\gamma$-ray rejection.

It is crucial that the selected neutron beam monitor fulfills the instrument requirement [8-11]. For instance, a beam monitor tested at NOP beamline in J-PARC showed a variation in the neutron detection efficiency which exceeds the tolerable level for high precision measurement of the neutron lifetime [12]. Different types of beam monitor will be used at ESS, some will be realized by ESS in kind partners [13-15] others will be manufactured by different suppliers. The aim of this work is to characterize seven 
neutron beam monitors, manufactured by several companies, and to compare their properties to be categorized for various applications.

\section{DETECTION TECHNIQUES}

Gas proportional counters have dominated the world of thermal neutron detection because of their robust design, high detection efficiency and low cost compared to solidstate detectors. Their operation is based on the absorption of thermal neutrons in the detector-converting medium. Lacking charge, thermal neutrons cannot interact with matter through Coulomb interaction, which is the dominant mechanism through which charged particles lose their energy. Therefore mechanisms for detecting thermal neutrons are based on indirect methods where neutrons interact with specific atomic nuclei to produce energetic ions. The most commonly used neutron interactions for thermal neutron detection are summarized in Table I.

The beam monitors under study are shown in Fig. 1. Their specifications are summarized in Table II. These monitors can be categorized depending on their design and the electronics they are equipped with, into two main categories: position sensitive and non-position sensitive. The multiwire proportional chambers (2D-MWPC) from Mirrotron [16] and the gas electron multiplier (2D-GEM) [17,18] from CDT [19] are position sensitive beam monitors, while other beam monitors are area-integrating, i.e., flux monitors.

All monitors studied here detect discrete neutron events, rather than an integral charge proportional to flux and all are sealed except the 2D-GEM monitor from CDT. This monitor should be filled with a gas mixture of $\mathrm{Ar} / \mathrm{CO} 2$ with a flow rate of 5-10 sccm. To determine the exact position of the neutron incidence, the detector includes position sensitive readout electrodes.

\section{A. Multiwire proportional chambers}

The monitors from Mirrotron and ORDELA are multiwire proportional chambers (MWPC) with low detection efficiency. Their entrance windows are thin aluminum membranes (around $1 \mathrm{~mm}$ thick) to minimize neutron attenuation. The two ORDELA models [20] are filled with ${ }^{3} \mathrm{He}+{ }^{4} \mathrm{He}+\mathrm{CF}_{4}$ and with ${ }^{14} \mathrm{~N}+\mathrm{CF}_{4}$ respectively, while the Mirrotron models are filled with ${ }^{3} \mathrm{He}+\mathrm{CF}_{4}$. The $\mathrm{CF}_{4}$

TABLE I. Nuclear reactions for thermal neutron detection.

\begin{tabular}{lccl}
\hline \hline Reaction & $\begin{array}{c}\text { Isotope } \\
\text { abundance }(\%)\end{array}$ & $\begin{array}{c}\text { Cross section (barn) } \\
\text { at } 1.8 \AA\end{array}$ & $\begin{array}{l}\text { Q-value } \\
(\mathrm{MeV})\end{array}$ \\
\hline${ }^{3} \mathrm{He}(\mathrm{n}, \mathrm{p}){ }^{3} \mathrm{H}$ & 0.014 & 5330 & 0.76 \\
${ }^{10} \mathrm{~B}(n, \alpha){ }^{7} \mathrm{Li}$ & 19.9 & 3838 & $2.31(94 \%)$ \\
& & & $2.79(6 \%)$ \\
${ }^{6} \mathrm{Li}(n, \alpha){ }^{3} \mathrm{H}$ & 7.6 & 947 & 4.78 \\
${ }^{14} \mathrm{~N}(\mathrm{n}, \mathrm{p}){ }^{14} \mathrm{C}$ & 99.63 & 1.91 & 0.62 \\
${ }^{235} \mathrm{U}(\mathrm{n}, 3 \mathrm{n}) \mathrm{ff}$ & 0.72 & 680.9 & $\approx 200$ \\
\hline \hline
\end{tabular}

gas is added as a stopping and quenching gas to limit the proton and triton ranges [21].

The 2D-MWPC from Mirrotron is a position sensitive monitor with a delay line readout. It is a chamber filled with a converter gas $\left({ }^{3} \mathrm{He}+\mathrm{CF}_{4}\right)$ for thermal neutron detection and consists of one anode frame and two cathode frames wired with gold-plated tungsten wire (1000 pixels). When a neutron is captured in the converter gas, electron-ion pairs are created. Then the electrons are accelerated toward the wire electrodes by the applied electric field. It is possible to determine the position of interaction by the integrated electronics. For this monitor the position encoding is based on delay lines [22]. The set of cathode wires is connected to a delay line read-out system. The delay time differences are measured for each set of cathodes with time-to-digital converters and these digital signals give an $\mathrm{XY}$ position encoded output. Beside the XY signals there is a summed anode signal which is used to obtain the pulse height spectra (PHS).

\section{B. Gas electron multiplier}

It is also possible to use a solid neutron converter with a gas readout. For the gas electron multiplier (GEM)-based beam monitor from CDT a $0.8 \mu$ m thick boron layer is used as a neutron converter layer. The thickness of the boron layer is chosen here for measurement purpose. However, a thinner layer will be selected for a full implementation. This layer is coated on the drift electrode. The charged products created ( $\alpha$ and triton) deposit their energy in the counting gas creating e-ion pairs along their track. The electrons then drift toward the GEM multiplier foil that is made of $50 \mu \mathrm{m}$ of Kapton substrate sandwiched between $5 \mu \mathrm{m}$ thick copper claddings on either side. The detector is supplied with its readout system. Each electrode of the readout structure is connected to one channel of a highly integrated ASIC, which contains for each channel a charge sensitive preamplifier, shaper, and discriminator.

\section{Scintillator}

Thermal neutron scintillation detectors often use lithium as the neutron converter. The principle of detection relies on the emission of light photons due to the interaction of radiation with the scintillation material [23]. Usually a photomultiplier is used to convert the light output of a scintillation pulse into a corresponding electrical signal. A scintillator detector from Quantum Detectors UK [24], consisting of a 2-dimensional array of neutron sensitive scintillator beads ( 35 beads each is $0.25 \mathrm{~mm}^{3}$ ) made of lithium silicate glass has been used in this work. The active area is $28 \times 42 \mathrm{~mm}^{2}$.

\section{Fission chamber}

Neutron-induced fission reactions can also be used for thermal neutron detection. In this case, extremely low 
(a)

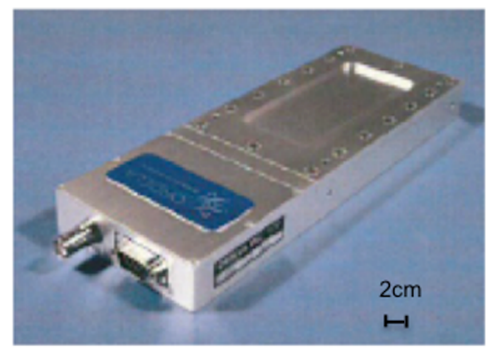

(b)

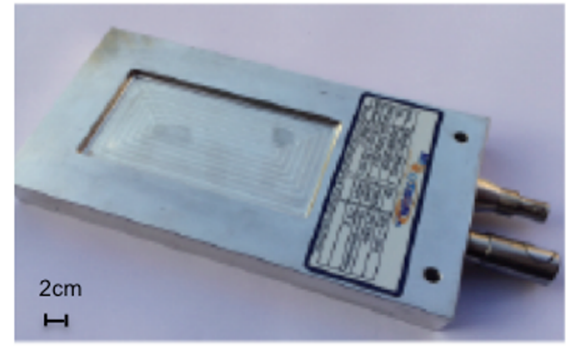

\section{Entrance window}

Entrance window

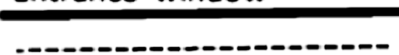

Counting gas

(d)
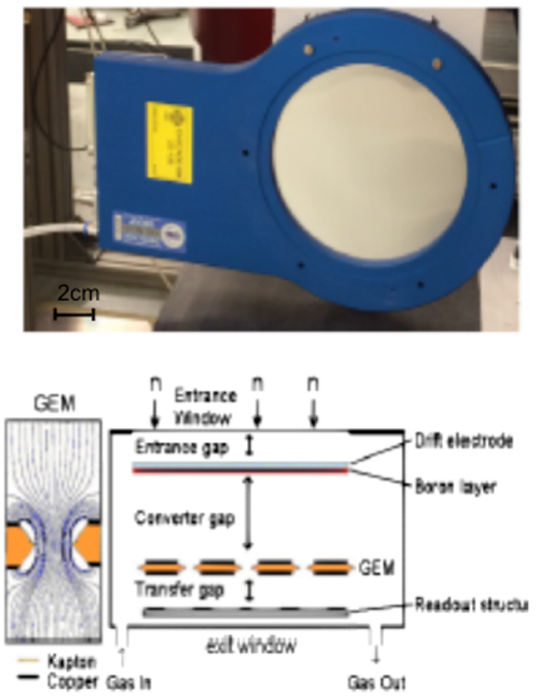

(c)
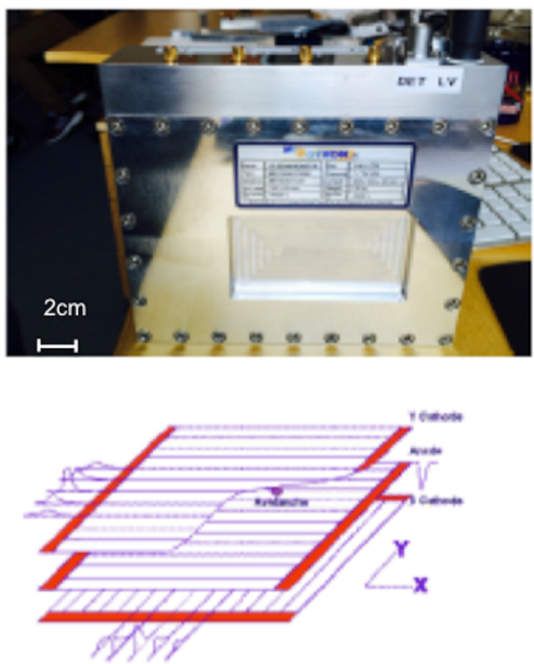

(f)
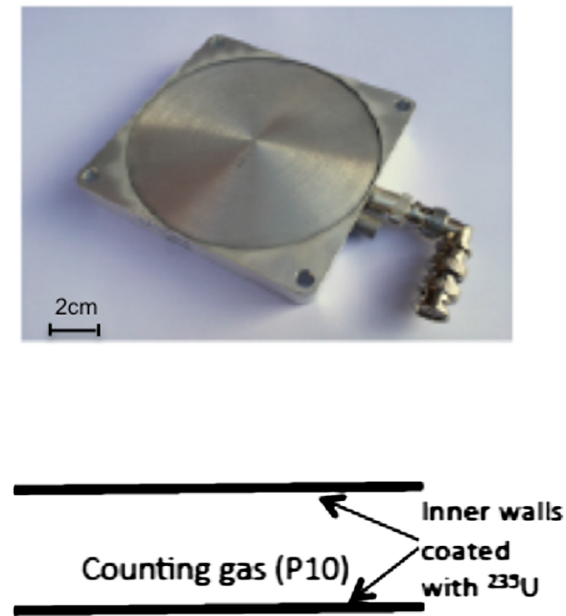

FIG. 1. Photographs and diagrams of the neutron beam monitors tested in this work: (a) MWPC from ORDELA, (b) MWPC from Mirrotron-0D, (c) MWPC from Mirrotron-2D, (d) 2D-GEM from CDT, e) scintillator from Quantum Detectors UK, (f) fission chamber from LND.

TABLE II. Specifications of the tested neutron beam monitors.

\begin{tabular}{|c|c|c|c|c|c|c|c|}
\hline Monitor Manufacturer & $\begin{array}{c}\text { MWPC } \\
\text { ORDELA }\end{array}$ & $\begin{array}{c}\text { MWPC } \\
\text { ORDELA }\end{array}$ & $\begin{array}{c}\text { MWPC } \\
\text { Mirrotron }\end{array}$ & $\begin{array}{c}\text { 2D-MWPC } \\
\text { Mirrotron }\end{array}$ & $\begin{array}{l}\text { 2D-GEM } \\
\text { CDT }\end{array}$ & $\begin{array}{c}\text { Scintillator } \\
\text { QD }\end{array}$ & $\begin{array}{c}\text { Fission chamber } \\
\text { LND }\end{array}$ \\
\hline active element & ${ }^{3} \mathrm{He}$ & ${ }^{14} \mathrm{~N}$ & ${ }^{3} \mathrm{He}$ & ${ }^{3} \mathrm{He}$ & ${ }^{10} \mathrm{~B}$ & ${ }^{6} \mathrm{Li}$ & ${ }^{235} \mathrm{U}$ \\
\hline partial pressure & 6.07 & 81.06 & 6.5 & 0.4 & $\cdots$ & $\cdots$ & \\
\hline total pressure (mbar) & $\ldots$ & $\ldots$ & $\ldots$ & $\ldots$ & 1000 &.. & 1013.2 \\
\hline filled gas & ${ }^{3} \mathrm{He}+{ }^{4} \mathrm{He}+\mathrm{CF}_{4}$ & $\mathrm{~N}+\mathrm{CF}_{4}$ & ${ }^{3} \mathrm{He}+\mathrm{CF}_{4}$ & ${ }^{3} \mathrm{He}+\mathrm{CF}_{4}$ & $\mathrm{Ar}+\mathrm{CO}_{2}$ & .. & $\mathrm{P} 10$ \\
\hline bias voltage $(\mathrm{V})$ & 850 & 850 & 1300 & $\begin{array}{c}\text { anode: }-3500 \\
\text { drift: } 1500\end{array}$ & -1000 & 650 & 300 \\
\hline active area $(\mathrm{mm})$ & $114 \times 51$ & $114 \times 51$ & $100 \times 50$ & $100 \times 50$ & $100 \times 100$ & $28 \times 42$ & $100 \times 100$ \\
\hline window thickness (mm) & 2 & 2 & 1 & 1 & 0.1 & 0.1 & 1 \\
\hline
\end{tabular}


background rates can be achieved thanks to the large $\mathrm{Q}$ value $(\approx 200 \mathrm{MeV})$ of the reaction. The most popular form of a fission detector is an ionization chamber coated with a layer of a fissile deposit such as ${ }^{235} \mathrm{U}$. Generally the fissionable materials used in a fission chamber are $\alpha$ emitters. Consequently, fission chambers have undesired pulse rates from the $\alpha$ background. This can be easily discriminated based on the pulse amplitude since the energy of an emitted $\alpha$ is 10 times smaller than the average energy of fission fragments. Typically the operating voltage of a fission chamber is between 50 and $300 \mathrm{~V}$. The principle of neutron detection in a fission chamber relies on the production of fission fragments that deposit their energy in the active volume, which is normally filled with argon. The pressure of the gas inside the chamber ensures that the range of fission fragments within the gas does not exceed the small dimensions of the detector. The fission chamber used in this work was purchased from LND [25]. It contains $13 \mathrm{mg}$ of ${ }^{235} \mathrm{U}$ and is filled with $\mathrm{P} 10$ gas $(90 \%$ argon and $10 \%$ methane). The detection depth is $19.1 \mathrm{~mm}$. Such type of monitor generates also fast neutrons and its use should be considered with care. Therefore, this type of monitor is recommended at only places sufficiently far away from the detector of the instrument.

\section{MEASUREMENTS METHODS}

The $\gamma$-ray sensitivity of a neutron beam monitor is an important criterion in its selection. This is because it is important to discriminate between the background radiation which is mainly $\gamma$ and the neutrons from the beam. To understand the degree of neutron to $\gamma$ discrimination, the beam monitors were tested using neutron and $\gamma$ sources at the Source Testing Facility, Lund University. The neutron source used is Be-based which emits neutrons up to about $11 \mathrm{MeV}$ with a maximum intensity in the $3-5 \mathrm{MeV}$ range [26]. In order to thermalize the emitted neutrons, the source is placed in a moderator made of polyethylene. For $\gamma$ sensitivity measurements different $\gamma$ sources $\left({ }^{60} \mathrm{Co},{ }^{57} \mathrm{Co}\right.$, ${ }^{133} \mathrm{Ba}$ ) at $2 \mathrm{~cm}$ from the monitor were used in order to evaluate the sensitivity of the monitors to low, medium and high-energy $\gamma$. The corresponding energies and intensities of these sources are summarized in Table III.

The $\gamma$ spectra was compared with the background measurement which means measurement in the absence of $\gamma$ and neutron sources. PHS are obtained as counts versus channel number. The channel numbers correspond to the energy deposited by the detected radiation in the active depth of each monitor. The PHS are normalized based on the activity of the sources used.

For measuring the ORDELA and Mirrotron monitors and the fission chamber we used the ORTEC 142-PC preamplifier. The preamplifier output was connected to a shaping amplifier. The output signal is sent to a multichannel analyzer to obtain the pulse height spectra. Even though
TABLE III. The $\gamma$ sources used with their corresponding energy and intensities.

\begin{tabular}{lccc}
\hline \hline Isotope & $\begin{array}{c}\text { Current activity } \\
(\mathrm{MBq})\end{array}$ & $\begin{array}{c}\text { Energy } \\
(\mathrm{keV})\end{array}$ & Intensity $(\%)$ \\
\hline${ }^{60} \mathrm{Co}$ & 15.58 & 1173.23 & 99.85 \\
& & 1332.48 & 99.98 \\
${ }^{57} \mathrm{Co}$ & 21.34 & 122 & 85.6 \\
& & 136 & 10.7 \\
${ }^{133} \mathrm{Ba}$ & 3.12 & 384 & 9 \\
& & 356 & 62 \\
& & 303 & 18 \\
& & 276 & 7 \\
& & 80 & 36 \\
& & $30-35$ & 117 \\
\hline \hline
\end{tabular}

this type of ORDELA monitor is supplied with preamplifier and discriminator, the anode signal was directly connected to the ORTEC 142-PC preamplifier for a more detailed analysis. The nominal high voltage (HV) up to $3500 \mathrm{~V}$ is provided by a HV power supply CAEN 1470 .

The scintillator beam monitor was supplied with its discriminator electronics. The discriminator processes the electrical signal producing two outputs: analogue and digital signal. The digital output appears whenever the signal amplitude is greater than the threshold. This is set so that the $\gamma$ part of the spectrum is rejected. However, for a more detailed analysis the analogue signal was used. In this case this signal is sent to the multichannel analyzer (MCA) to obtain the pulse height spectra. The measuring time is 10 minutes unless stated otherwise.

For efficiency measurements we used the R2D2 beam-line at the JEEPII reactor at IFE, Norway. This beam comes from the reactor through a shutter as shown in Fig. 2. The collimator in the shutter is made of Cd layers and has a $36^{\circ}$ divergence. The beam is then monochromated by a composite $\mathrm{Ge}$ wafer monochromator. The beam size at the tested monitors is controlled by two JJ X-Ray IB-C80-AIR slits with borated aluminum blades which are placed before the monitors. The expected neutron flux at the center of the beam is of the order of $10^{5} \mathrm{n} / \mathrm{s} \mathrm{cm}^{2}$ thermal neutrons. The wavelength of the neutron beam can be adjusted from $0.88 \AA$ to $2.4 \AA$ by the rotation of the monochromator. For high statistics, all the measurements were performed at $2.4 \AA$.

Efficiency was measured using a 1-inch stainless steel ${ }^{3}$ He-tube (Tube RSP40810227 manufactured by GE-Reuter Stokes). The tube was operated at a ${ }^{3} \mathrm{He}$ pressure of $10 \mathrm{~atm}$, and a voltage of $1400 \mathrm{~V}$. The walls of the tube are made of stainless steel and have a thickness of $0.508 \mathrm{~mm}$. The efficiency setup used is shown in Fig. 3, where both the Hetube and the monitor are irradiated with the same beam successively. It is important to make sure that the beam is narrow enough so that it is fully measured by the He-tube and its intensity is adjusted so no saturation occurs in the tube. For the beam monitors with very low detection 

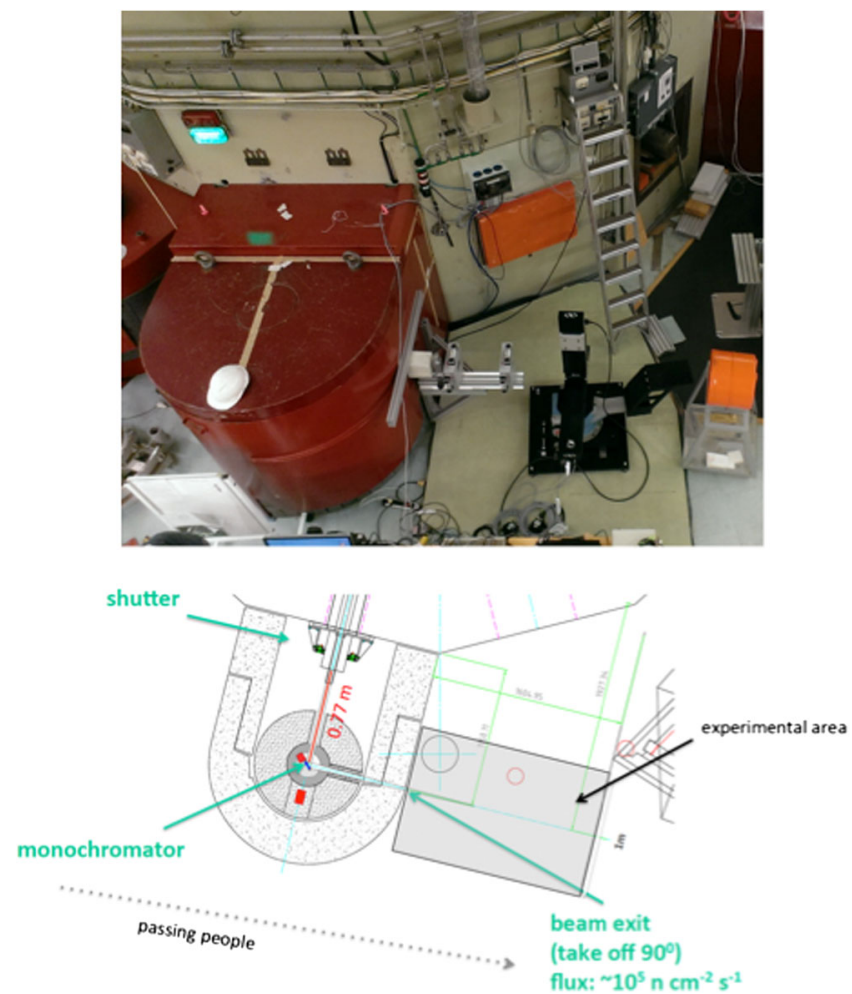

FIG. 2. The setup used for thermal neutron detection at R2D2 beam line at IFE-Norway.

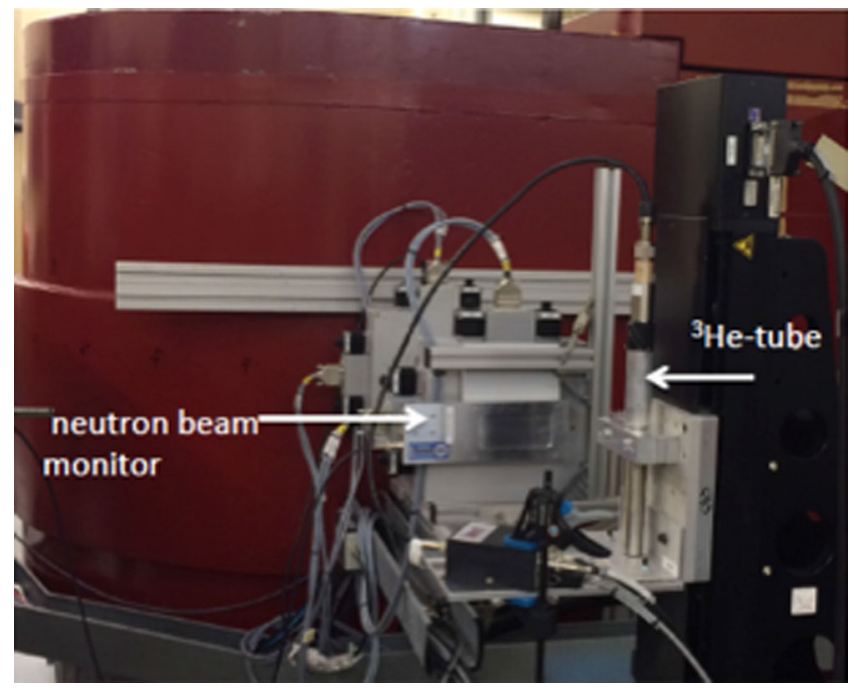

FIG. 3. Setup used at R2D2 beam for efficiency and attenuation measurements.

efficiency it is necessary to measure for a long time to accumulate enough statistics.

\section{GAMMA SENSITIVITY}

The PHS obtained by all the monitors except the GEM-2D are shown in Figs. 4-9. A rough energy

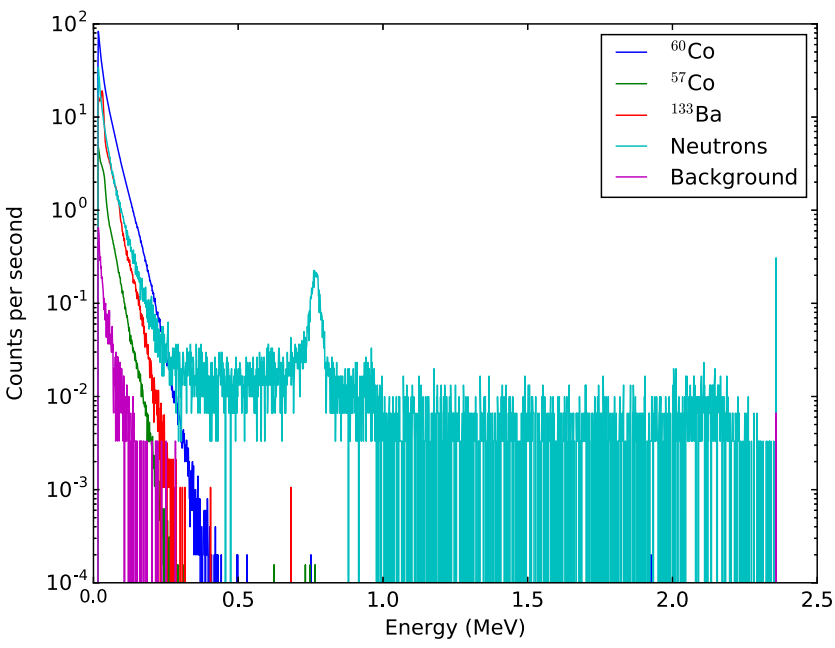

FIG. 4. Pulse height spectra measured by the Mirrotron monitor using a neutron source and using three $\gamma$ sources separately $\left({ }^{60} \mathrm{Co},{ }^{57} \mathrm{Co},{ }^{133} \mathrm{Ba}\right)$.

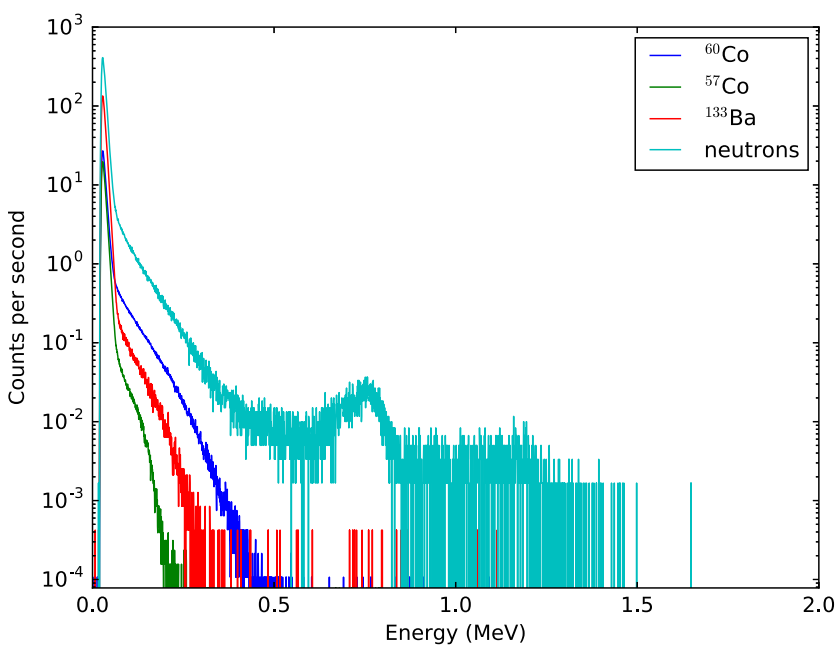

FIG. 5. Pulse height spectra measured by the Mirrotron-2D monitor using a neutron source and using three $\gamma$ sources separately $\left({ }^{60} \mathrm{Co},{ }^{57} \mathrm{Co},{ }^{133} \mathrm{Ba}\right)$.

calibration of the PHS was performed based on the peak position in each spectra. The PHS obtained by the Mirrotron monitor (BM-100X50) filled with ${ }^{3} \mathrm{He}+\mathrm{CF}_{4}$ are shown Fig. 4. Neutron detection results in a peak at the high energy, while $\gamma$ contributes to the low energy part. There is a valley between the $\gamma$ and neutron detection which allows $\gamma$ discrimination. As the energy of $\gamma$ increases, a higher energy deposition is obtained in all the monitors. This is seen by a greater energy deposited by the ${ }^{60} \mathrm{Co}$ compared to ${ }^{133} \mathrm{Ba}$ and ${ }^{57} \mathrm{Co}$ as shown in Figs. 4-9.

Having the same converting gas $\left({ }^{3} \mathrm{He}\right)$, the PHS features of the Mirrotron, Mirrotron-2D, and the ORDELA-He look similar. However, the ORDELA produces a much 


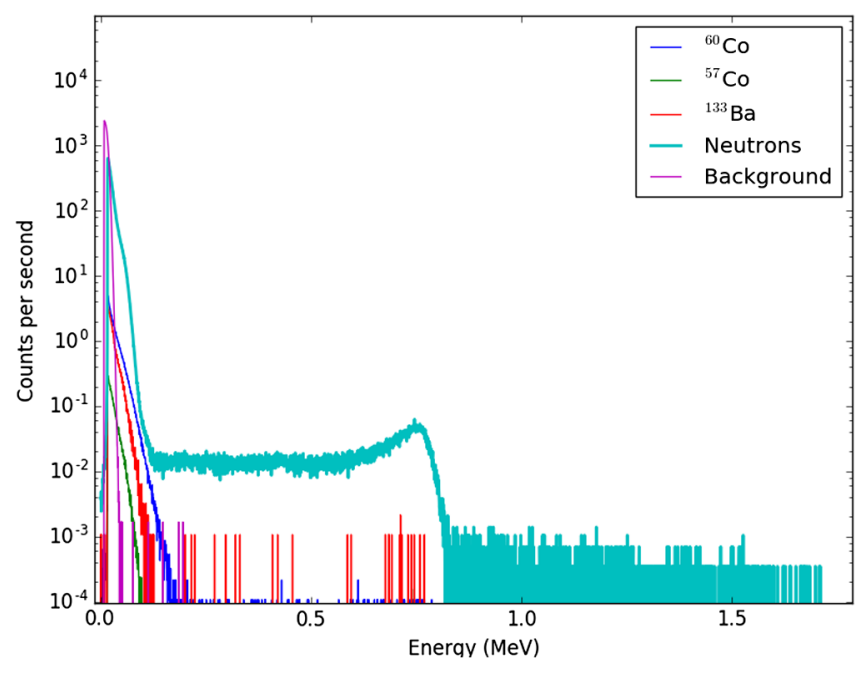

FIG. 6. Pulse height spectra measured by the ORDELA-He monitor using a neutron source and using three $\gamma$ sources separately $\left({ }^{60} \mathrm{Co},{ }^{57} \mathrm{Co},{ }^{133} \mathrm{Ba}\right)$.

clearer and sharper edge, while the Mirrotron has a clearer narrow peak.

Nitrogen can also be used as a neutron converter gas when very low neutron detection efficiency is required. The neutron detection is based on charged particles created from ${ }^{14} \mathrm{~N}(\mathrm{n}, \mathrm{p}){ }^{14} \mathrm{C}$ reaction. Due to the low cross section of $\mathrm{N}$ with neutrons and the low efficiency of this monitor longer time measurements ( 22 hours) were required to obtain adequate statistics. As shown in Fig. 7, the neutron detection is revealed by the presence of the peak at higher energy around $0.6 \mathrm{MeV}$. $\gamma$ detection is revealed by the counts from all the sources in to the low-energy region as shown in Fig. 7.

The PHS of the scintillator monitor is shown in Fig. 8. It is clear that the $\gamma$ region extends from low energy up to

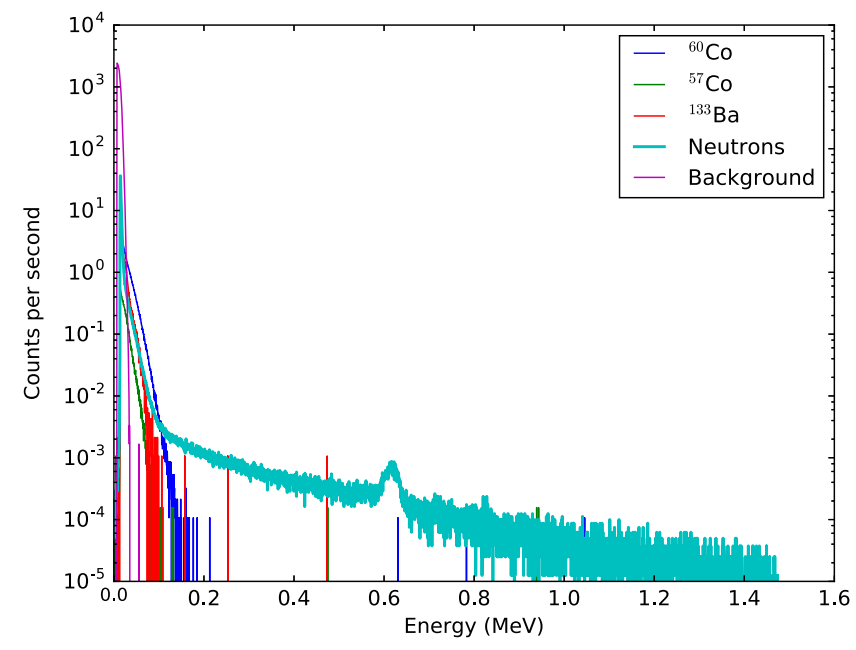

FIG. 7. Pulse height spectra measured by the ORDELA-N monitor using a neutron source and using three $\gamma$ sources separately $\left({ }^{60} \mathrm{Co},{ }^{57} \mathrm{Co},{ }^{133} \mathrm{Ba}\right)$.

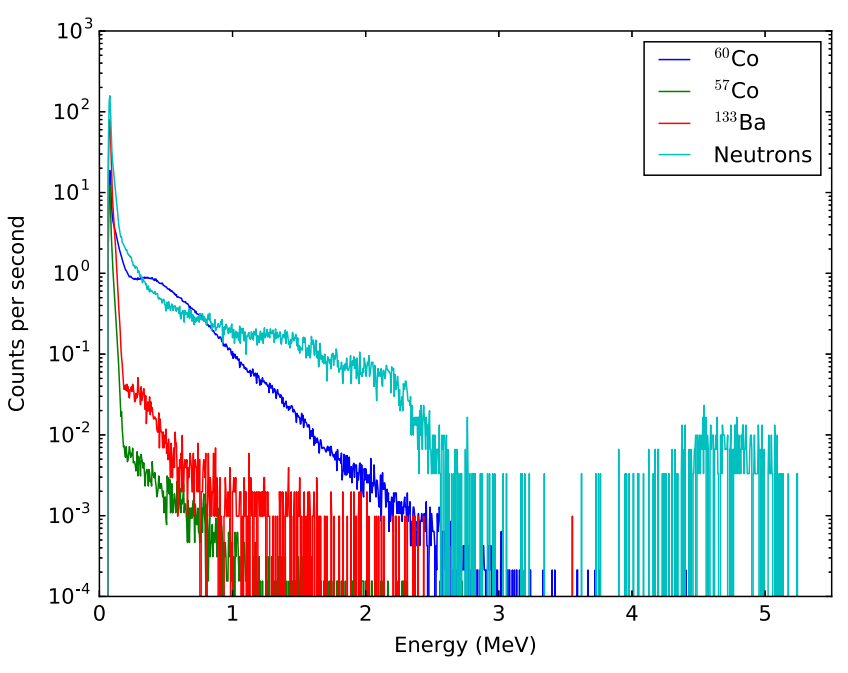

FIG. 8. Pulse height spectra measured by the scintillator monitor using a neutron source and using three $\gamma$ sources separately $\left({ }^{60} \mathrm{Co},{ }^{57} \mathrm{Co},{ }^{133} \mathrm{Ba}\right)$.

$3 \mathrm{MeV}$. The neutron peak is well resolved at higher energy which allows a good discrimination between $\gamma$ and neutrons. The neutron detection is revealed by the presence of a neutron peak around 4.7 MeV.

On the other hand, due to the high Q value $(\approx 200 \mathrm{MeV})$, the fission chamber with ${ }^{235} \mathrm{U}$ as a detection medium shows excellent $\gamma$ discrimination as shown in Fig. 9. No $\gamma$ signals from any source could be detected in the fission chamber with the experiment used. All the spectra are identical in the low-energy region. This part of the spectrum comes from $\alpha$ decays of ${ }^{235} \mathrm{U}$ which are few up to $10 \mathrm{MeV}$. Thus a very clean discrimination can be accomplished using fission chambers.

It was not possible to obtain for the PHS for the ${ }^{10} \mathrm{~B}$-based 2D-GEM monitor from CDT, based on the

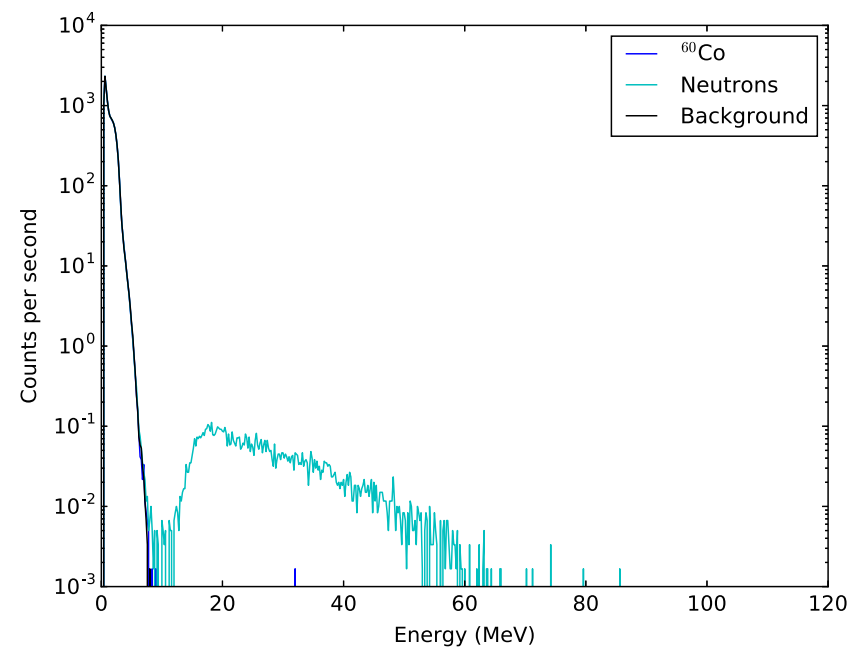

FIG. 9. Pulse height spectra measured by the LND fission chamber using a neutron source and using ${ }^{60} \mathrm{Co}$. 


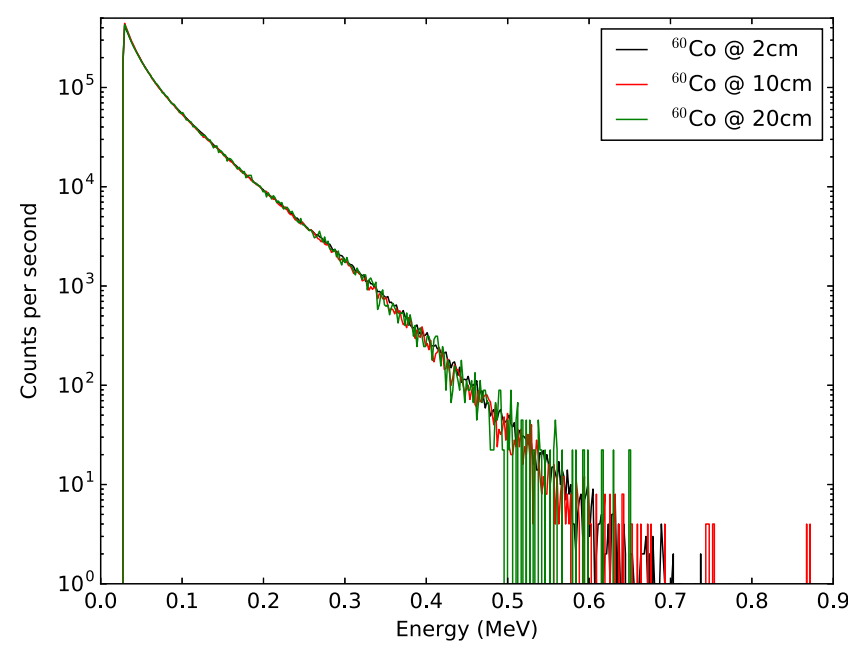

FIG. 10. Pulse height spectra measured by the Mirrotron monitor using ${ }^{60} \mathrm{Co}$ at three distances.

electronics provided. Thus a 2D-image was used for the $\gamma$ sensitivity estimation, which showed no measurable $\gamma$-ray detection even when the monitor was tested at the lowest threshold level using a ${ }^{60} \mathrm{Co}$ source. This could be attributed to the threshold level which is already set in a way to reject the $\gamma$. This $\gamma$ insensitivity has also been observed in other GEM-based neutron detectors [15].

To better understand if there is a pile-up effect in these monitors, they have been measured at several distances with respect to the $\gamma$ source used. Figure 10 is a an example of this study. The PHS are normalized with respect to their corresponding solid angle. It is shown that with the $\gamma$ sources used there is no significant effect.

Figure 11 shows the $\gamma$ and neutron counts from the measurements where ${ }^{60} \mathrm{Co}$ and Be-based neutron sources were used respectively. These counts are collected in 10 minutes as a function of the threshold level. $\gamma$ and neutron counts decrease with the increase in the threshold level. For lower $\gamma$ sensitivity the threshold level should be set in a region which maximizes neutron counts and minimizes $\gamma$ counts. This differs from one monitor to another based on the monitor $\gamma$ sensitivity. A correctly chosen threshold level allows a high $\gamma$ rejection [27]. For instance the fission chamber shows the lowest $\gamma$ sensitivity where the region of high $\gamma$ counts is very narrow allowing high neutron counts with negligible $\gamma$ counts.

\section{NEUTRON DETECTION}

Figures 12-15 show the PHS of the ORDELA and Mirrotron beam monitors using the neutron beamline at IFE and background when the beam is off. The neutron detection is revealed by the neutron peak in all the spectra. From such spectra information regarding efficiency was extracted. The neutron detection is revealed in all the
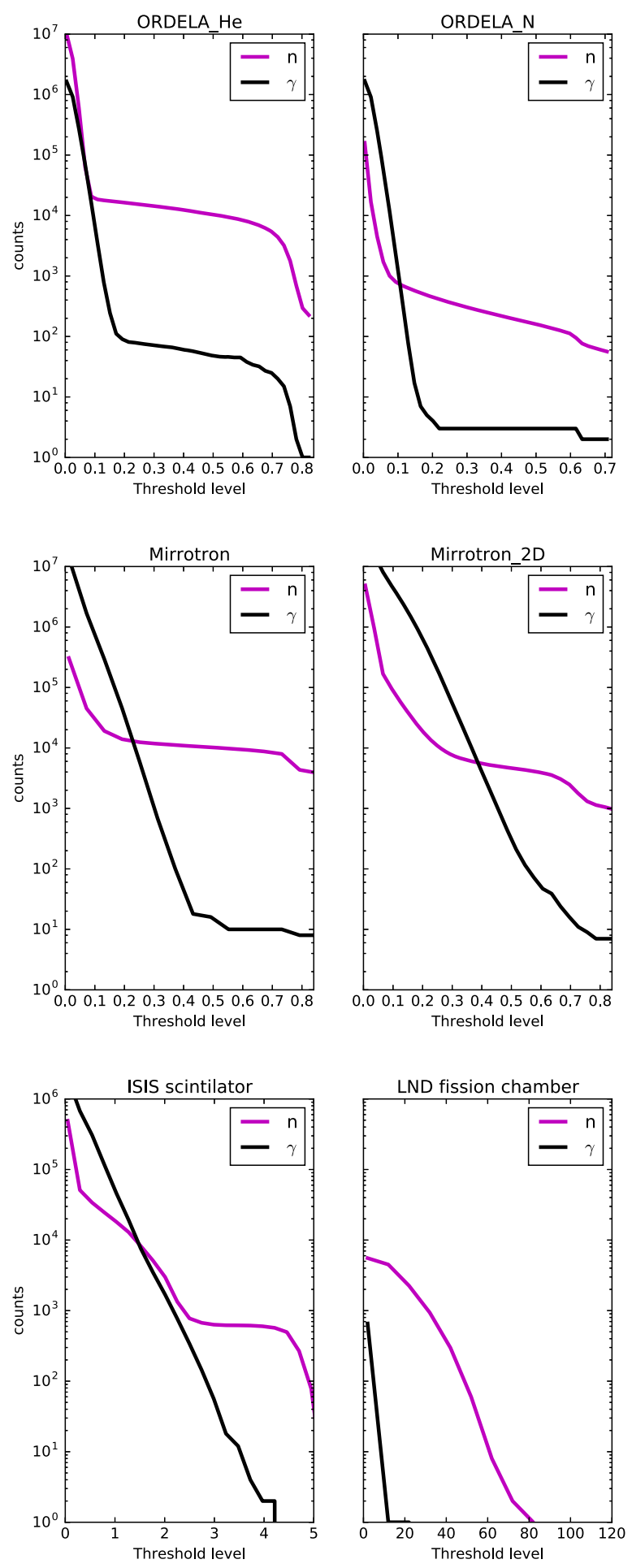

FIG. 11. $\quad \gamma$ and neutron counts using ${ }^{60} \mathrm{Co}$ and Be-based neutron sources respectively. These counts are collected in $10 \mathrm{~min}$ as a function of the set threshold level which represents the energy $(\mathrm{MeV})$ in the PHS.

spectra by the presence of a peak while $\gamma$ counts contribute to the lower-energy region. In the Mirrotron and the ORDELA-He monitors the peak is around the $760 \mathrm{keV}$ while for the ORDELA-N the peak is at $620 \mathrm{keV}$. 


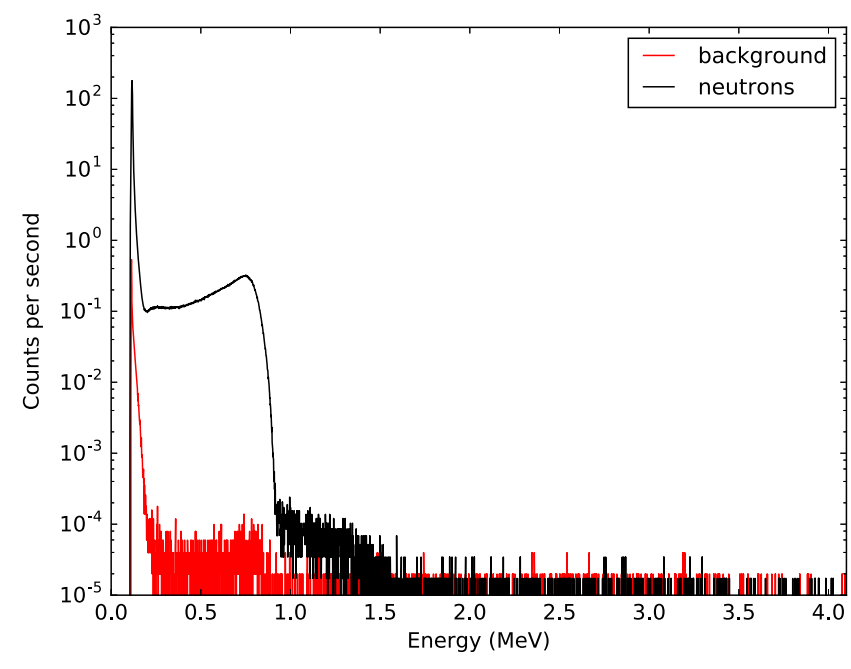

FIG. 12. Pulse height spectra measured by the ORDELA-He monitor using neutron beam line at $2.4 \AA$.

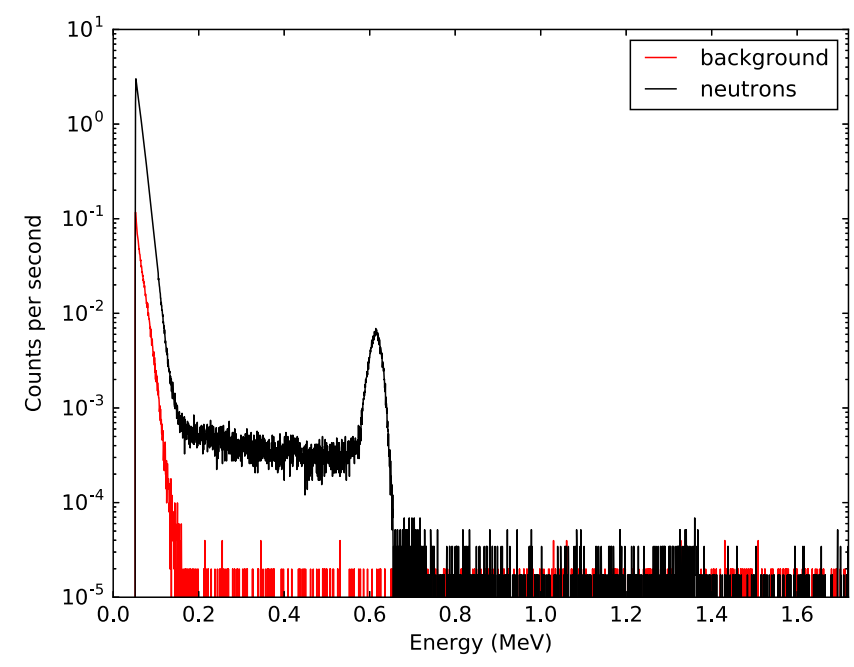

FIG. 13. Pulse height spectra measured by the ORDELA-N monitor using neutron beam line at $2.4 \AA$.

\section{A. Efficiency, attenuation, and scattering measurements}

The efficiency of each monitor is measured by dividing the total number of counts above the noise level by the total number of neutrons in the beam, which is estimated from the total counts in the ${ }^{3} \mathrm{He}$-tube ( $96 \%$ efficiency). The efficiency has been evaluated at a $\lambda=2.4 \AA$. These results are summarized in Table IV. Whilst it is important to continuously monitor the neutron beam at each instrument, it is critical that the beam monitor used does not significantly affect the beam. Since there is always a probability of absorbing or scattering a neutron by the monitor and its housing material it is important to study their corresponding attenuation coefficients. This is illustrated in Fig. 16 where the transmitted neutron flux $(\mathrm{T})$ is equal to the

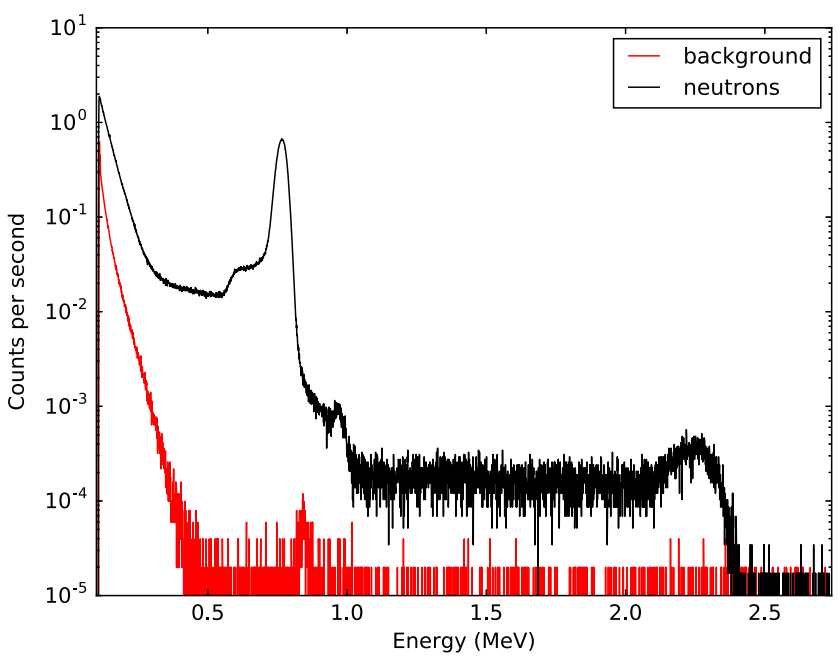

FIG. 14. Pulse height spectra measured by the Mirrotron monitor using neutron beam line at $2.4 \AA$.

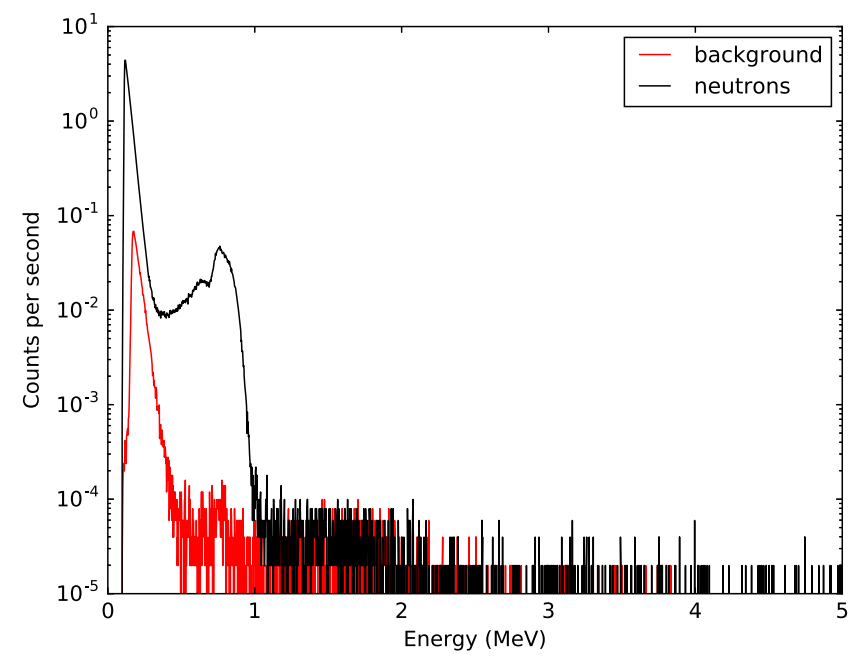

FIG. 15. Pulse height spectra measured by the Mirrotron-2D monitor using neutron beam line at $2.4 \AA$.

incident flux $(\mathrm{F})$ minus the attenuated neutrons which is due to scattering $(\mathrm{S})$ and absorption (A).

$$
\begin{aligned}
T & =F-A-S \\
T / F & =\text { transmission factor }
\end{aligned}
$$

For these measurements the same setup as for the efficiency measurements was used, except that the monitor was placed in front of and removed from the ${ }^{3} \mathrm{He}$ tube in order to measure loss of counts due to the monitor. This allows the fraction of neutrons attenuated by the monitor to be determined. Assuming scattering by each monitor is isotropic, the scattered neutrons were measured by a ${ }^{6} \mathrm{Li}$-glass scintillator detector from SCIONIX. This 


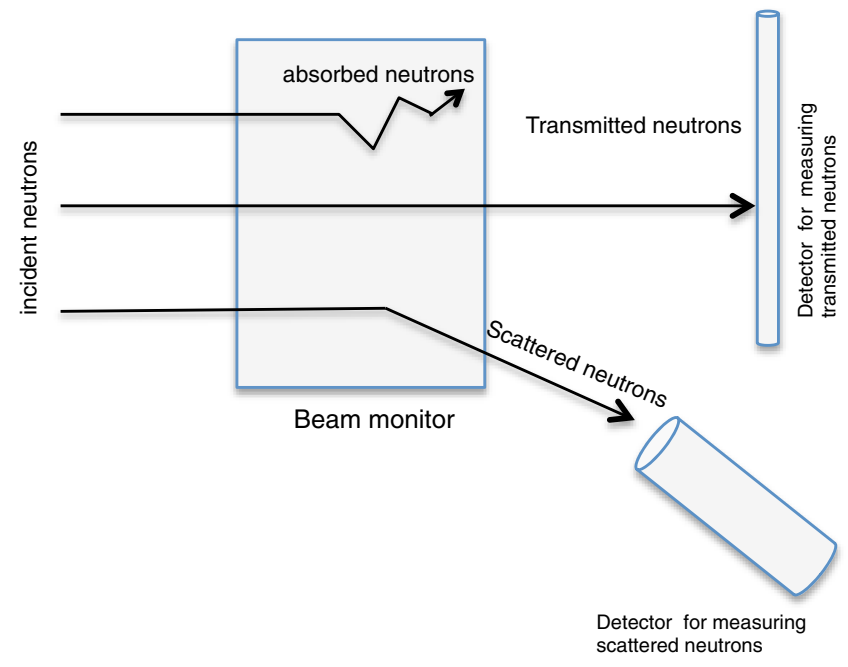

FIG. 16. Schematic presentation showing the setup used for measuring transmission and scattering of neutrons, the detector used for transmission is a ${ }^{3} \mathrm{He}$-tube and a ${ }^{6} \mathrm{Li}$-glass scintillator from SCIONIX is used for measuring scattering.

detector has sufficient scintillator thickness to stop at least $99 \%$ of incoming neutrons at $1.8 \AA$. We will assume for the analysis here, that this is the efficiency of the detector. The beam stability was monitored by a monitor from Canberra while performing these measurements to ensure the stability of the neutron beam. The total attenuation, efficiency and scattering percentages are summarized in Table IV. The statistical error is relatively low compared to the measured attenuation and efficiency percentages. For scattering measurement the main source of standard deviation is due to the solid angle calculation. These values are summarized in Table IV.

As the 2D-GEM monitor from CDT has a relatively thick layer $(0.8 \mu \mathrm{m})$ of boron, it shows the highest efficiency. The relatively high efficiency of this monitor is chosen to reduce the measuring time. This also increases absorption however in a realistic implementation, a much thinner layer would be chosen to reduce attenuation. The ORDELA monitors show different efficiencies depending on the quantity and cross section of the gas used. For instance the ${ }^{14} \mathrm{~N}$ cross section is several orders of magnitude lower than ${ }^{3} \mathrm{He}$ making the latter more sensitive to neutron detection. The ORDELA and Mirrotron monitors filled with ${ }^{3} \mathrm{He}$ have similar parameters and comparable efficiencies. Relatively high efficiency monitors of each type have been selected to expedite the measurement times. The ORDELA-N and scintillator monitors show measured efficiencies higher than the ones declared by the supplier. This could be explained by a higher quantity of the neutron sensitive material than the declared one. The measured efficiency has been compared to the calculated efficiency based on the supplier information regarding the gas pressure and the depth of the counting volume. This calculation is based on a simple consideration of cross sections. The equation used for calculating the efficiency for the MWPC monitors is given below:

$$
\varepsilon=1-\exp (-\mu \lambda . \rho x)
$$

where $\varepsilon$ is the efficiency, $\mu$ is the gas absorption factor for the detector gas $(\operatorname{atm} \mathrm{cm} \lambda)^{-1}, \lambda$ is the wavelength in $\AA$, $\rho$ is the absolute counting gas pressure, and $\mathrm{x}$ is the depth of the counting volume.

The high level of attenuation shown by all the monitors except the scintillator monitor is striking. For instance, for the ORDELA monitors the neutron beam has to cross the $2 \mathrm{~mm} \mathrm{Al}$ window on both sides. The Mirrotron-2D showed three times higher attenuation compared to the Mirrotron monitor. This could be attributed to the thicker window and the Kapton foil used inside the detector. This 2D beam monitor type could be used for beam diagnostic purposes removing it from the neutron beam when it is not in use. The scintillator monitor shows the lowest attenuation percentage. This could be explained by a thinner aluminum window $(100 \mu \mathrm{m})$. The LND depth is $19.1 \mathrm{~mm}$ and is coated with $13 \mathrm{mg}$ of ${ }^{235} \mathrm{U}$. It attenuates the beam by $3.8 \%$. The difference between the measured and the calculated attenuation in the LND fission chamber may be attributed to the way the uranium is coated on the Al plate where additional materials might be used to make the adhesion possible. The ORDELA monitors attenuates the beam by

TABLE IV. The measured efficiency and attenuation by all the monitors using the R2D2 beam-line at $2.4 \AA$.

\begin{tabular}{lcccccr}
\hline \hline Monitor & $\begin{array}{c}\text { Measured } \\
\text { efficiency at } \\
2.4 \AA(\%)\end{array}$ & $\begin{array}{c}\text { Calculated } \\
\text { efficiency at } \\
2.4 \AA(\%)\end{array}$ & $\begin{array}{c}\text { Supplier } \\
\text { efficiency at } \\
1.8 \AA(\%)\end{array}$ & $\begin{array}{c}\text { Measured } \\
\text { attenuation at } \\
2.4 \AA(\%)\end{array}$ & $\begin{array}{c}\text { Calculated } \\
\text { attenuation at } \\
2.4 \AA(\%)\end{array}$ & $\begin{array}{c}\text { Measured } \\
\text { scattering at } \\
2.4 \AA(\%)\end{array}$ \\
\hline ORDELA-He & 0.12 & 0.13 & 0.1 & 4.5 & 4.05 & $3.9 \pm 0.4$ \\
ORDELA-N & $2.1 \times 10^{-3}$ & $1.3 \times 10^{-3}$ & $1 \times 10^{-3}$ & 4.4 & 4.05 & $3.8 \pm 0.4$ \\
Mirrotron & 0.11 & 0.13 & 0.1 & 2.5 & 2.05 & $4 \pm 0.9$ \\
Mirrotron- 2D & $1.5 \times 10^{-2}$ & $1.7 \times 10^{-2}$ & $1.5 \times 10^{-2}$ & 7.3 & 4.5 & $9 \pm 1$ \\
CDT & 3 & 4 & 3 & 10.6 & 6.9 & $10.3 \pm 0.7$ \\
Scintillator & 0.052 & 0.012 & 0.01 & 0.49 & 0.1 & $0.74 \pm 0.2$ \\
Fission chamber & 0.01 & 0.021 & 0.01 & 3.87 & 2.4 & $3.8 \pm 0.3$ \\
\hline \hline
\end{tabular}




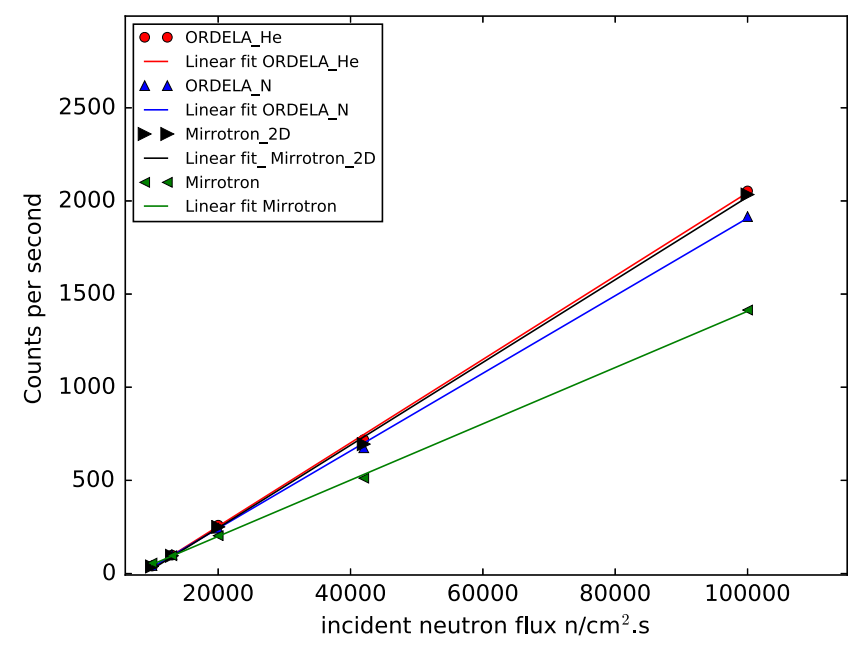

FIG. 17. Neutron counts measured by different monitors versus the variation in the incident neutron flux. Counts from ORDELA-N and Mirrotron-2D monitors were multiplied by a factor of 100 and 10 , respectively.

about $4 \%$ which is due to the $2 \mathrm{~mm} \mathrm{Al}$ window thickness on both sides. The high attenuation factor in most of the monitors is mainly due to the thickness of the window. Thinner Al windows (less than $1 \mathrm{~mm}$ ) should be used for lower attenuation effects.

\section{B. Linearity}

It is important that a beam monitor measures the rate in the neutron beam with a high precision. Normally the response of a beam monitor should be linearly correlated to a change in the neutron beam. For studying the linearity response of these monitors as function of the change in the

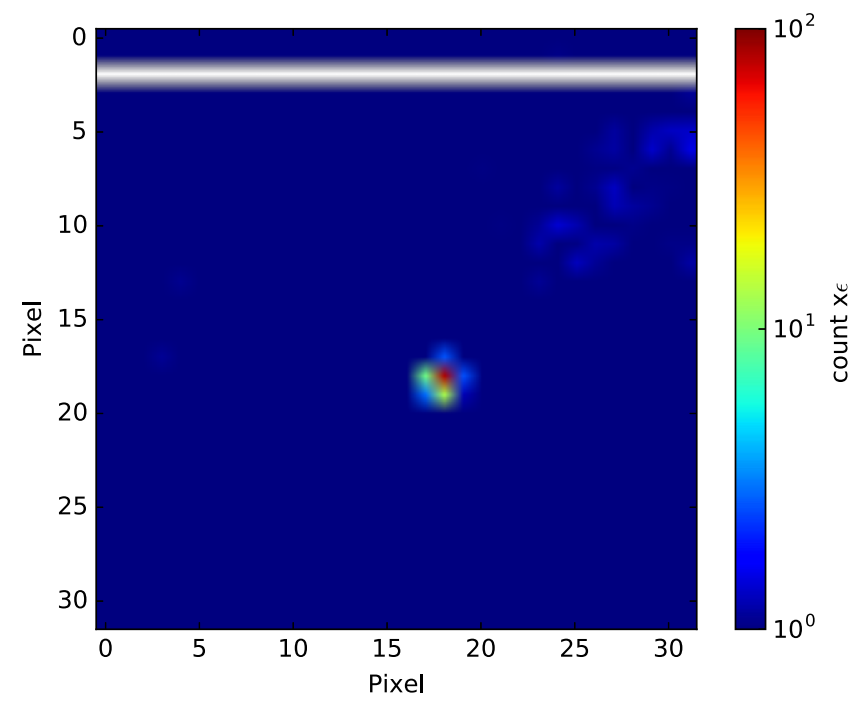

FIG. 18. Two-dimensional image measured by GEM monitor at R2D2 at IFE using a neutron beam size of $1 \times 1 \mathrm{~mm}^{2}$ at $2.4 \AA$. The white strip is a dead electronics channel. One pixel size is $3.12 \mathrm{~mm}$.

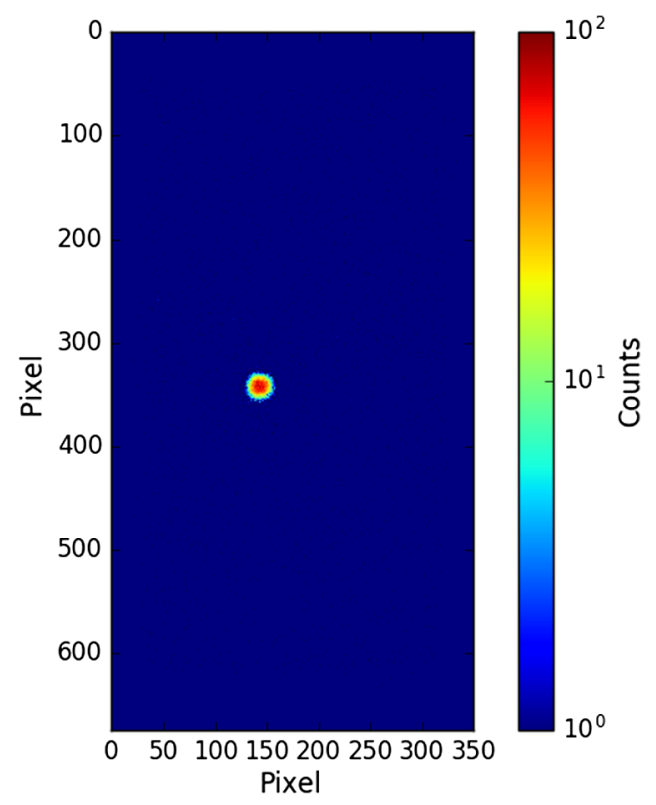

FIG. 19. Two-dimensional image measured by Mirrotron-2D at R2D2 at IFE using a neutron beam size of $1 \times 1 \mathrm{~mm}^{2}$ at $2.4 \AA$. The pixel size is $1 \mathrm{~mm} \times 1 \mathrm{~mm}$.

beam flux, several plastic plates were used. Each plate has a thickness of around $3 \mathrm{~mm}$. The plates were placed close to the beam-line exit and $1 \mathrm{~m}$ away from the beam monitor and used as neutron attenuators. The attenuation in the neutron intensity by using several plates was measured by the ${ }^{3} \mathrm{He}$-tube. The beam is attenuated by about $50 \%$ by using one plate.

The neutron counts from different beam monitors as function of the change in the incoming neutron flux are shown in Fig. 17. The results show that there is no measurable deviation from linearity up to the used flux $\left(10^{5} \mathrm{n} / \mathrm{cm}^{2} \mathrm{~s}\right)$.

\section{Position resolution}

Finally, one important characteristics of a position sensitive monitor is its spatial resolution defined as FWHM (full width at half maximum). For such characterization the beam should be as narrow as possible. This was achieved using a collimated beam defined with two sets of slits. This makes sure that the beam is not widened due to divergence. The measuring time was 1 hour for the GEM monitor and 18 hours for the Mirrotron-2D. The estimated FWHM value of the GEM is $3.1 \mathrm{~mm}$ and $1.3 \mathrm{~mm}$ for Mirrotron-2D. The 2D-image measured by the 2DGEM and Mirrotron-2D monitors are shown in figures 18 and 19 respectively. The 2D-image for the 2D-GEM is presented as counts multiplied by its efficiency.

\section{CONCLUSIONS}

Different types of neutron beam monitors (MWPC, GEM, scintillators and fission chamber) purchased from different companies (ORDELA, Mirrotron, Scintillator 
from Quantum Detectors, LND and CDT) have been tested using a neutron source and different $\gamma$ sources and using the neutron R2D2 beamline at IFE-Norway. The efficiency, attenuation and gamma sensitivity have been studied. It is striking the high level of attenuation shown by all but one of the monitors. This is related to the used materials in each monitor (mainly the $\mathrm{Al}$ window or kapton in case of the used position sensitive monitors). For instance this factor could be reduced by reducing the window thickness. The fission chamber from LND showed the lowest gamma sensitivity thanks to the large Q value $(\approx 200 \mathrm{MeV})$ of the neutron-induced fission reaction. At the moment, the monitor efficiency is far lower than the beam attenuation. This work provides useful information for users at different facilities in order to choose a proper monitor which best satisfies their requirements. Based on these measurements some of these beam monitors will be customized in order to match the ESS instrument requirements. Future developments on monitors should focus on reducing the level of scattering and attenuation of the beam.

\section{ACKNOWLEDGMENTS}

This work is funded by EU Horizon 2020 framework, BrightnESS project 676548 and was carried out in part at the Source Testing Facility, Lund University. We would like to thank K. Berry, W. Schweika, and N. Rhodes for the useful discussions; the manufacturers for their openness.

[1] ILL, http://www.ill.eu.

[2] ISIS, http://www.isis.stfc.ac.uk.

[3] SNS, http://neutrons.ornl.gov/sns.

[4] J-PARC, http://j-parc.jp/index-e.html.

[5] ESS web site, https://europeanspallationsource.se.

[6] S. Peggs et al., European spallation source technical design report. Technical report, 2013.

[7] R. Hall-Wilton and C. Theroine, Status of the European Spallation Source ESS AB, the instrument selection process, and a fundamental physics beamline at the ESS, Phys. Procedia 51, 8 (2014).

[8] J. E. Bateman, N. J. Rhodes, and R. Stephenson, A highresolution, imaging neutron beam monitor using gas microstrip technology, Nucl. Instrum. Methods Phys. Res., Sect. A 477, 365 (2002).

[9] A. Pietropaolo, G. Verona Rinati, C. Verona, E. M. Schooneveld, M. Angelone, and M. Pillon, A single-crystal diamond-based thermal neutron beam monitor for instruments at pulsed neutron sources, Nucl. Instrum. Methods Phys. Res., Sect. A 610, 677 (2009).

[10] P. M. De Lurgio, R. T Klann, C. L. Fink, D. S. McGregor, P. Thiyagarajan, and I. Naday, A neutron detector to monitor the intensity of transmitted neutrons for small-angle neutron scattering instruments, Nucl. Instrum. Methods Phys. Res., Sect. A 505, 46 (2003).

[11] A. V. Belushkin, A. A. Bogdzel, V. V. Zhuravlev, T. T. Panteleev, L. Y. Jai, A. N. Chernikov, A. V. Churakov, and V. N. Shvetsov, Two-coordinate position-sensitive monitordetector of thermal neutrons, Tech. Phys. 53, 117 (2008).

[12] T. Ino, H. Otono, K. Mishima, and T. Yamad, Precision neutron flux measurement with a neutron beam monitor, J. Phys. Conf. Ser. 528, 012039 (2014).

[13] G. Croci, C. Cazzaniga, G. Claps, M. Tardocchi, M. Rebai, F. Murtas, E. Vassallo, R. Caniello, E. P. Cippo, G. Grosso, V. Rigato, and G. Gorini, Characterization of a thermal neutron beam monitor based on gas electron multiplier technology, Prog. Theor. Exp. Phys. 2014, 83H01 (2014).

[14] G. Albani, E. P. Cippo, G. Croci, A. Muraro, E. Schooneveld, A. Scherillo, R. Hall-Wilton, K. Kanaki, C. Höglund, L. Hultman et al., Evolution in boron-based gem detectors for diffraction measurements: from planar to 3d converters, Meas. Sci. Technol. 27, 115902 (2016).

[15] G. Croci, G. Claps, R. Caniello, C. Cazzaniga, G. Grosso, F. Murtas, M. Tardocchi, E. Vassallo, G. Gorini, C. Horstmann et al., Gem-based thermal neutron beam monitors for spallation sources, Nucl. Instrum. Methods Phys. Res., Sect. A 732, 217 (2013).

[16] Mirrotron, http://www.mirrotron.kfkipark.hu/.

[17] F. Sauli, Gaseous Radiation Detectors: Fundamentals and Applications (Cambridge University Press, Cambridge, England, 2014), Vol. 36.

[18] F. Sauli, Gem: A new concept for electron amplification in gas detectors, Nucl. Instrum. Methods Phys. Res., Sect. A 386, 531 (1997).

[19] CDT GmbH. CASCADE detector technologies, http://ncdt.com/.

[20] ORDELA inc., http://www.ordela.com/.

[21] M. K. Kopp, K. H. Valentine, L. G. Christophorou, and J. G. Carter, New gas mixture improves performance of 3 he neutron counters, Nucl. Instrum. Methods Phys. Res. 201, 395 (1982).

[22] J. Orbán, Serial delay-line readout for position sensitive neutron beam monitor, Pollack Periodica 10, 53 (2015).

[23] N. J Rhodes, E. M Schooneveld, and R. S Eccleston, Current status and future directions of position sensitive neutron detectors at $\{$ ISIS $\}$, Nucl. Instrum. Methods Phys. Res., Sect. A 529, 243 (2004).

[24] Quantum detectors Ltd., http://quantumdetectors.com/ neutrons/.

[25] LND inc., http://www.Indinc.com/products/category/43/.

[26] J. Scherzinger, R. A. Jebali, J. Annand, K. Fissum, R. HallWilton, S. Koufigar, N. Mauritzson, F. Messi, H. Perrey, and E. Rofors, A comparison of untagged gamma-ray and tagged-neutron yields from 241 ambe and 238pube sources, arXiv:1611.00213.

[27] A. Khaplanov, F. Piscitelli, J-C Buffet, J-F Clergeau, J. Correa, P. van Esch, M. Ferraton, B. Guerard, and R. HallWilton, Investigation of gamma-ray sensitivity of neutron detectors based on thin converter films, J. Instrum. 8, P10025 (2013). 